\title{
Estimating Young's Modulus of Single-Walled Zirconia Nanotubes Using Nonlinear Finite Element Modeling
}

\author{
Ibrahim Dauda Muhammad, ${ }^{1}$ Mokhtar Awang, ${ }^{1}$ Othman Mamat, ${ }^{1}$ and Ku Zilati Ku Shaari ${ }^{2}$ \\ ${ }^{1}$ Department of Mechanical Engineering, Universiti Teknologi PETRONAS, 31750 Tronoh, Perak, Malaysia \\ ${ }^{2}$ Department of Chemical Engineering, Universiti Teknologi PETRONAS, 31750 Tronoh, Perak, Malaysia \\ Correspondence should be addressed to Ibrahim Dauda Muhammad; ibrahimuhd@yahoo.com
}

Received 17 October 2014; Revised 13 December 2014; Accepted 15 December 2014

Academic Editor: Shiren Wang

Copyright (c) 2015 Ibrahim Dauda Muhammad et al. This is an open access article distributed under the Creative Commons Attribution License, which permits unrestricted use, distribution, and reproduction in any medium, provided the original work is properly cited.

\begin{abstract}
The single-walled zirconia nanotube is structurally modeled and its Young's modulus is valued by using the finite element approach. The nanotube was assumed to be a frame-like structure with bonds between atoms regarded as beam elements. The properties of the beam required for input into the finite element analysis were computed by connecting energy equivalence between molecular and continuum mechanics. Simulation was conducted by applying axial tensile strain on one end of the nanotube while the other end was fixed and the corresponding reaction force recorded to compute Young's modulus. It was found out that Young's modulus of zirconia nanotubes is significantly affected by some geometrical parameters such as chirality, diameter, thickness, and length. The obtained values of Young's modulus for a certain range of diameters are in agreement with what was obtained in the few experiments that have been conducted so far. This study was conducted on the cubic phase of zirconia having armchair and zigzag configuration. The optimal diameter and thickness were obtained, which will assist in designing and fabricating bulk nanostructured components containing zirconia nanotubes for various applications.
\end{abstract}

\section{Introduction}

Zirconia $\left(\mathrm{ZrO}_{2}\right)$ is considered to be among the most important ceramic materials owing to its exceptional mechanical properties together with its stability at high temperatures [1]. It is used as a refractory in insulation, for metal coating, also as abrasives, enamels, and glazes, and as support material for catalysts [2], and due to its ion conductivity it is used in oxygen pumps for partial regulation [3], gas sensors [4], and high temperature fuel cells [5]. Also, $\mathrm{ZrO}_{2}$ is considered as one of the most radiation-resistant ceramics, thus having specific application in the nuclear industry [6].

At atmospheric pressure, $\mathrm{ZrO}_{2}$ has three phases or polymorphs. At high temperatures (above $2350^{\circ} \mathrm{C}$ ), $\mathrm{ZrO}_{2}$ exists as cubic fluorite structure $(\mathrm{fm} 3 \mathrm{~m})$, while at low temperatures (below $1150^{\circ} \mathrm{C}$ ), monoclinic baddeleyite $(P 211 C)$ structure dominates. A tetragonal phase exists at intermediate phase having P42/nmc symmetry [7]. Most applications of $\mathrm{ZrO}_{2}$ are based on its cubic polymorph which can be stabilized by doping with oxides such as $\mathrm{CaO}, \mathrm{MgO}, \mathrm{CeO}_{2}$, and $\mathrm{Y}_{2} \mathrm{O}_{3}$ [8]. Improved results are obtained by reducing the crystallite size to few $\mathrm{nm}$, with an average of about $15 \mathrm{~nm}$ [9]; this results in $\mathrm{ZrO}_{2}$ nanomaterials in form of dots, slabs, sheets, and tubes at atomic scale level. For instance, $\mathrm{ZrO}_{2}$ is presently being tested as catalyst support for several reactions and displays a much higher activity than some other oxides [10]. Thus, for some years now, progress has been attained on the synthesis and study of various nanostructure materials containing zirconia. Significant consideration has been given to $\mathrm{ZrO}_{2}$ nanotube (ZNT) due to its existing and potential applications, such as components of oxygen sensors, host matrix for optical functional materials, and electrolytes in solid-oxide fuel cells [11].

Studies have shown that nanotubes have mechanical properties far superior when compared to that of bulk material [4]. These novel nanotubes (NTs) are projected to have high stiffness, wear resistance, strength, lower thermal conductivity, and high melting temperature [2] and are 
still retaining high plasticity because of their nearly infinite length-to-width ratio [12]. These higher material properties render such nanotubes suitable for a range of applications [11].

Progress in scanning probe methods, especially atomic force microscope (AFM), has assisted in providing novel settings to estimate some mechanical properties of nanotubes [12]. Calculations and interpretations of physical properties of ZNT and similar materials most often are conducted using AFM or electron microscopes: the scanning electron microscope (SEM) or the transmission electron microscope (TEM). Possibly the most common instrument in characterization of these materials is AFM [14], which applies very small forces $(\mathrm{nN})$ and detects tiny displacements $(\mathrm{nm})$. Several experimental investigations on the mechanical properties have been conducted for carbon nanotubes [15-17] and inorganic nanotubes [18] with Young's modulus of ZNT detected experimentally to be between 30 and $52 \mathrm{GPa}$ using nanoindentation setup [19].

However, several difficulties and challenges have been experienced during the mechanical characterization of nanotubes due to their tiny size and high cost of required equipment [14]. In addition, there are other problems related to specimen collection, handling, and setting and quantifying small forces and small deformations. Precise setting of the load application spot and analysis of the results further complicate the procedure [15].

Owing to some problems encountered during experimental analysis, theoretical modelling methods have been used recently to evaluate mechanical properties of NTs. Amongst the existing modelling techniques, the molecular dynamics (MD) method has been used widely [20] and is focused on the force field and total potential energy linked to the interatomic potentials of nanotubes. Based on this approach, the bonding and nonbonding potentials are represented in relation to the force constants and the distance change amongst the atomic bonds, and then elastic moduli are estimated by using different small-strain deformation modes [21]. But MD simulation is not effective for timeconsuming or fixed problem(s); thus, it has limitation in the study of the mechanical properties of nanotubes. The other method is the continuum or finite element method [22], whereby nanotubes are made up of elements regarded as beams or shells that are subjected to bending, tension, or torsional loading. This facilitates the nanotube to be modelled as a shell-like or frame structure and the mechanical behaviour realized by finite element method or classical continuum mechanics [23].

The magnitude of axial Young's modulus for carbon nanotubes (CNT) has been found out by simulation to be in the range of $1.0 \mathrm{TPa}$ to $5.5 \mathrm{TPa}[20,24]$ with that of $\mathrm{WS}_{2}, \mathrm{MoS}_{2}$, and $\mathrm{TiO}_{2}$ nanotubes to be $143 \mathrm{GPa}, 230 \mathrm{GPa}$, and $270 \mathrm{GPa}$, respectively [22, 23]. Much information is required on the mechanical behaviour of $\mathrm{ZNT}$, as sufficient study has not been done experimentally and numerically [25]. Therefore, the purpose of this paper is to simulate the mechanical behaviour of ZNT when exposed to axial tension in order to estimate Young's modulus using finite element (FE) approach.

\section{Modeling and Simulation}

2.1. Molecular Mechanics. Based on the concept of molecular mechanics, the total potential energy $(U)$ is specified as sum of specific potential constituents as a result of interactions [21, 23, 24]:

$$
U=U_{\rho}+U_{\theta}+U_{\omega}+U_{\tau}+U_{\mathrm{vdw}}+U_{\mathrm{es}}
$$

where $U_{\rho}, U_{\theta}, U_{\tau}$, and $U_{\omega}$ are energies related to bond stretching, bond-angle inversion, and bond torsion and inversion, respectively, and are based on bonding, while $U_{\mathrm{vdw}}$ and $U_{\mathrm{es}}$ are van der Waals and electrostatics interactions, respectively, and are not based on bonding. The energy terms in (1) can be expressed using different functions depending on the loading condition and type of material [26]. For the $\mathrm{Zr}-\mathrm{O}$ bond in ZNT the ionic bonding dominates; thus, the significant parts of the potential energy are $U_{\rho}$ and $U_{\text {es }}$ and are represented by Buckingham and Coulomb expressions, respectively. For $\mathrm{ZrO}_{2}$, the potential energy is expressed as a sum of two-body interactions of the form [21]

$$
U=A \exp \left(\frac{-r_{i j}}{\rho}\right)-\frac{C}{r_{i j}^{6}}+\frac{q_{i} q_{j}}{4 \pi \varepsilon_{o} r_{i j}},
$$

where $A, \rho$, and $C$ are constants describing the contributions of short-range interaction of each particular pair and $r_{i j}$ is the distance between $\mathrm{Zr}$ and $\mathrm{O}_{2}$ atoms. Also $q_{i}$ and $q_{j}$ represent the charges on the pairs of ions and $\varepsilon_{o}$ is the permittivity of free space.

The concept of energy equivalence can be used to link the force factors in molecular mechanics and the element stiffness in structural mechanics $[15,20,22-24,26]$ which will allow simulation of the mechanical behavior of ZNT. Based on Timoshenko's theory of elasticity for beams, the relations between the beam strain energies and the harmonic potentials are expressed as [27]

$$
E=\frac{4 K_{i j} r_{i j}}{\pi d^{2}}, \quad G=\frac{32 K_{i j k l} r_{i j}}{\pi d^{2}},
$$

where $E$ and $G$ represent Young's modulus and shear modulus, respectively, for $\mathrm{Zr}-\mathrm{O}$ bond in form of beam with $d$ as diameter and $r_{i j}$ as length. Also, $K_{i j}$ and $K_{i j k l}$ denote the stretching force and bending force constants, respectively.

The diameter, which is equivalent to thickness, and the Poisson ratio for the $\mathrm{Zr}-\mathrm{O}$ structural bond element can be determined using analytical, mechanistic, or numerical models [26].

\subsection{Finite Element Modeling (FEM)}

2.2.1. Structure. The geometry of inorganic nanotubes is built on the similar models used for CNT where the tubes are supposed to be made by rolling up of nanosheets (NNS) to form a hollow cylinder and may be single- or multiwalled. For CNT, the basic structural unit is a single atomic layer, known as graphene [28]. The nanotube is defined by the translation vector $\mathbf{L}=l_{1} \mathbf{a}+l_{2} \mathbf{b}$ and the chiral vector, $\mathbf{R}=n \mathbf{a}_{1}+m \mathbf{a}_{2}$, $\left(l_{1}, l_{2}, n\right.$, and $m$ are integers, and $\mathbf{a}_{\mathbf{1}}$ and $\mathbf{a}_{2}$ are translation 

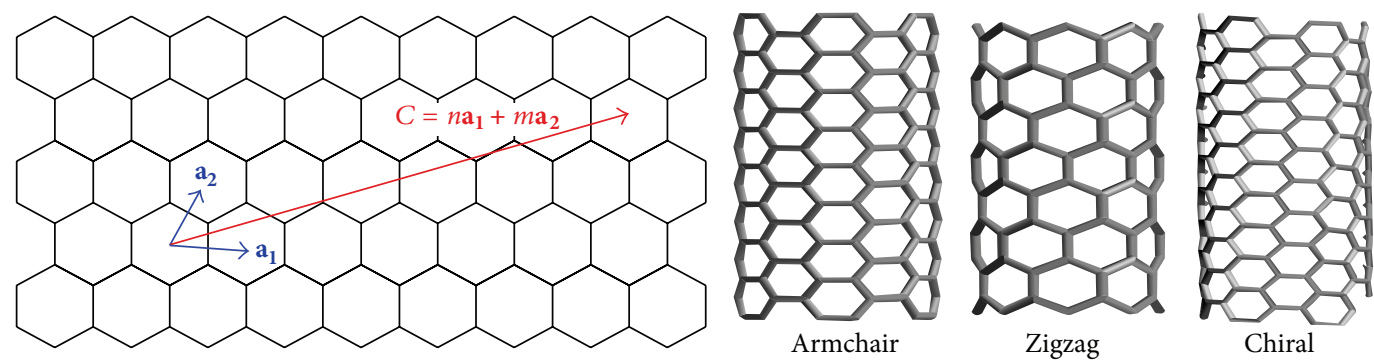

FIGURE 1: Schematic representation of the relation between nanosheet and nanotubes [13].

TABLE 1: Computed constants for interactions of pairs of atoms in $\mathrm{ZrO}_{2}[21]$.

\begin{tabular}{lccc}
\hline Pair $i j$ & $A i j(\mathrm{eV})$ & $\rho i j(\AA)$ & $C\left(\mathrm{eV} \AA^{6}\right)$ \\
\hline $\mathrm{Zr}-\mathrm{O}$ & 985.87 & 0.3760 & 0.0 \\
$\mathrm{O}-\mathrm{O}$ & 22764.3 & 0.1490 & 27.89 \\
$\mathrm{Zr}-\mathrm{O}$ & 0.0 & 0.0 & 0.0 \\
\hline
\end{tabular}

vectors of the $2 \mathrm{D}$ lattice) as shown in Figure 1 . The nanotube of the chirality $(n, m)$ is achieved by folding the layer in a manner that the chiral vector $\mathbf{R}$ becomes the circumference of the nanotube. The orthogonality relations $(\mathbf{R L})=0$ are used to define the NT chirality $(n, m)$ harmonious with the initial 2D lattice periodicity [25]. By designation $(n, n)$ is armchair, $(n, 0)$ is zigzag, and $(n, m)$ is chiral [29].

From analysis conducted on other nanotubes [20, 22-28], the circumference length $(L)$ of the chiral vector $\left(C_{h}\right)$ and diameter of the single-walled ZNT are expressed as

$$
\begin{aligned}
& L=\left|C_{h}\right|=a \sqrt{n^{2}+m^{2}+n m,} \\
& d=\frac{L}{\pi}=\frac{a}{\pi} \sqrt{n^{2}+m^{2}+n m},
\end{aligned}
$$

where $a$ is the lattice constant of $\mathrm{ZrO}_{2}$ and is related to the $\mathrm{Zr}-\mathrm{O}$ bond length. Using first-principles calculations, $a$ and $\mathrm{Zr}-\mathrm{O}$ bond length were found out to be 5.0755 and $2.1953 \AA$, respectively [30].

2.2.2. Modelling. Based on available geometrical parameters for ZNT [25, 30], single-walled ZNT having different dimensions was developed by means of Surface Builder in Material Studio software for armchair and zigzag types. Each of the structures was saved as PDB file and the atomic coordinates and connectivity data in the produced file for the nanotube were obtained using a coding from Python Wing IDE [31]. Thereafter, a macro was written to model the ZNT in ANSYS with the atoms and bonds regarded as the nodes and elements, respectively.

For the modelling of the ZNT bonds, the 3D BEAM188 element is used. The BEAM188 element is appropriate for investigating thin to relatively thick beam assemblies. This element is founded on Timoshenko beam theory. Shear distortion influences are built-in. BEAM188 may be regarded as a 2-mode linear beam element having six degrees of freedom at all nodes. The degrees of freedom at all nodes

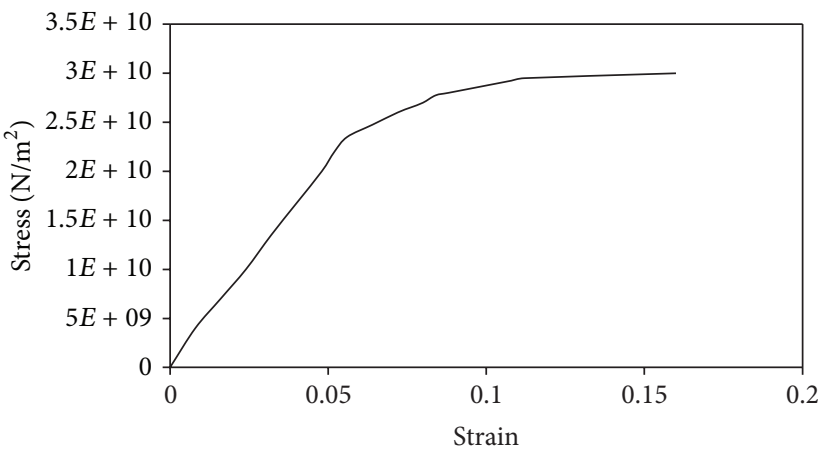

FIGURE 2: Stress-strain curve for $\mathrm{Zr}-\mathrm{O}$ bond as element.

consist of translations in $x, y$, and $z$ orientations and cycles or rotations about the $x, y$, and $z$ orientations. Distortion of the cross-sections is presumed to be unrestricted. The beam elements are suitable for use where linear, nonlinear, sizeable rotation, and/or significant strain occurs [32]. The diameter and Poisson ratio for the element ( $\mathrm{Zr}-\mathrm{O}$ bond) were determined to be $0.018 \mathrm{~nm}$ and 0.1897 , respectively, using CrystalMaker and CASTEP [30]. By differentiating the potential energy expression in (2), the effective force in the bond or element is obtained as

$$
F=-\frac{A \exp ^{(-r / \rho)}}{\rho}-\frac{6 C}{r^{7}}-\frac{q_{i} q_{j}}{4 \pi \varepsilon_{o} r^{2}} .
$$

For zirconia, $\varepsilon_{o}=0.55263614 \times 10^{-12} \mathrm{C}^{2} \mathrm{eV}^{-1} \AA^{-1}, q_{i}=$ $q_{\mathrm{Zr}}=4 e$, and $q_{j}=q_{\mathrm{O}}=-2 e$, where $e$ is magnitude of electronic charge, $1.602 \times 10^{-19} \mathrm{C}$, and the parameters of pairs of interactions of atoms in $\mathrm{ZrO}_{2}$ are presented in Table 1 [21].

For $\mathrm{Zr}-\mathrm{O}$ bond, the relationship between stress and strain is shown in Figure 2 taking element's cross-sectional area to be $2.545 \times 10^{-20} \mathrm{~m}^{2}$. The $\mathrm{Zr}-\mathrm{O}$ bond displays nonlinear or rate-dependent stress-strain behaviour due to large strain during deformation $[26,27]$. The problem due to large strain is reduced by using Multilinear Isotropic Hardening Material Model (MISO) for the element.

From the curve in Figure 2, Young's modulus of the $\mathrm{Zr}-$ O element was computed to be $5.01 \times 10^{11} \mathrm{~Pa}(501 \mathrm{GPa})$, representing the slope in the linear region, and the value is similar to $491 \mathrm{GPa}$ that was obtained for bulk $\mathrm{ZrO}_{2}$ by firstprinciple calculations using CASTEP [30]. 


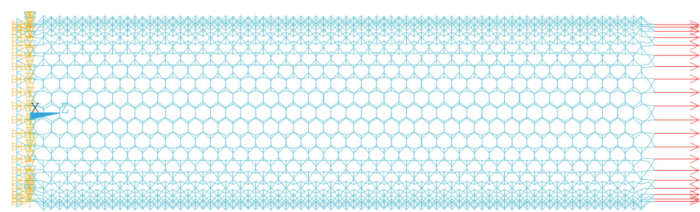

(a)

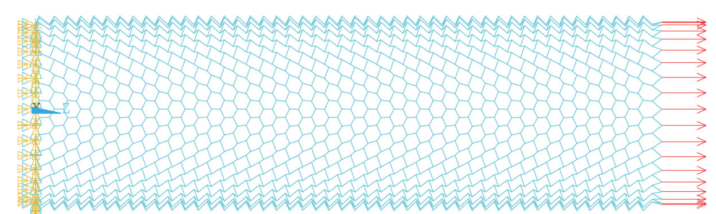

(b)

FIGURE 3: FE models with boundary conditions for $(a)$ armchair $(20,20)$ and (b) zigzag $(35 \times 0)$ SWZNT.
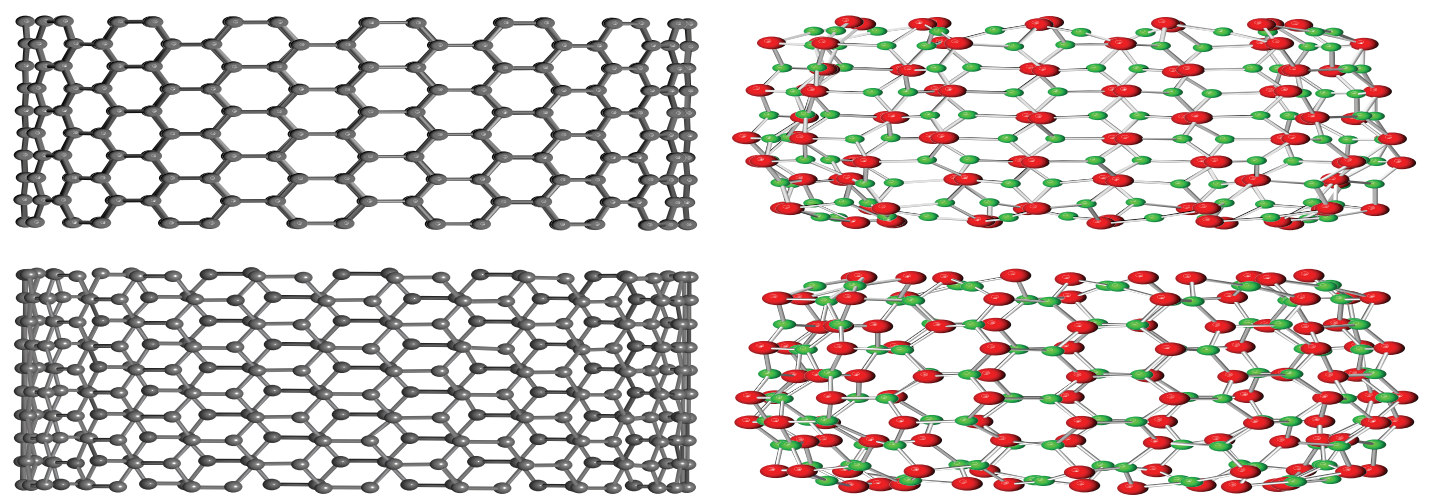

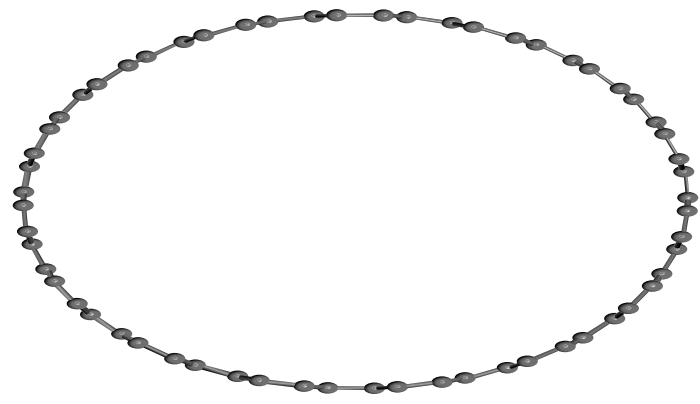

(a)

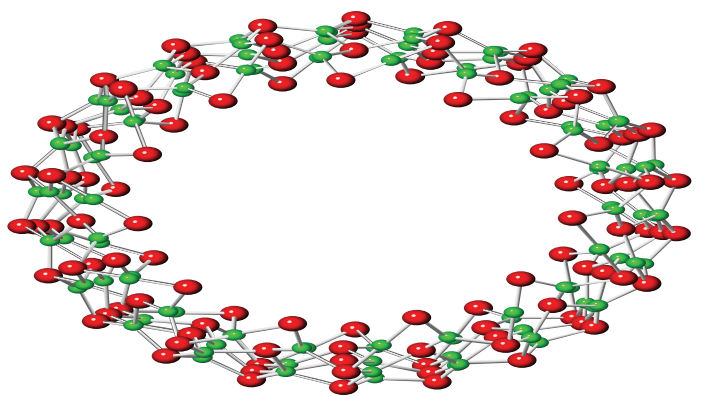

(b)

Figure 4: Front, side, and top views for (a) $15 \times 15 \mathrm{CNT}$ and (b) $11 \times 11 \mathrm{ZNT}$.

2.2.3. Boundary Conditions. Two main types of SWZNTs, armchair and zigzag, were considered. A fixed displacement was applied at one end and prescribed displacement was applied at the other end axially as depicted in Figure 3. Due to the applied displacement, resultant reaction force $(F)$ occurs and is obtained after nonlinear simulation of forces in all the nodes. The procedure is repeated by changing the applied displacements for different nanotubes having different configurations.

Young's modulus $(E)$ for each nanotube after simulation was determined using classical elasticity theory [20]:

$$
E=\frac{\sigma}{\varepsilon}=\frac{F / A_{t}}{\Delta L_{t} / L_{t}}=\frac{F L_{t}}{\pi d t \Delta L_{t}},
$$

where $F$ is the resultant force on nodes at the applied end, $A_{t}=\pi d t$ is the cross-sectional area of the nanotube having $d$ as the diameter and $t$ as thickness, and $L_{t}$ and $\Delta L_{t}$ are the initial length and elongation, respectively, in the axial direction.
In order to improve the accuracy of the simulated results, the nanotubes having the maximum $E$ with minimum diameter were selected and mesh convergence study is conducted by increasing the division of each element from 1 to $2,3,5,7$, and 10 .

\section{Results and Discussion}

The geometrical parameters of ZNT in relation to diameter and length depend on chirality, with chiral type having the highest size followed by the zigzag and then armchair. The difference between two similar nanotubes in relation to length and diameter is $1.7: 1: 2.6$ for armchair, zigzag, and chiral types, respectively. The variations are similar to that of boron nitride nanotubes (BNNT) and CNT $[33,34]$, but the values are less than that of ZNT which have longer and thicker atomic bond. Details of the geometrical parameters of some ZNTs are stated in Table 2.

The symmetry of the SWZNT is not uniform compared to CNT. The orientation depends on the chirality, with armchair having more uniform diameter across the tube and zigzag 
TABLE 2: Geometrical parameters for modeled ZNTs.

\begin{tabular}{lcccccc}
\hline Chirality $(n \times m)$ & Diameter $(\AA)$ & Length $(\AA)$ & Atoms/nodes & Bonds/elements & \multicolumn{2}{c}{ Bond length $(\AA)$} \\
Maximum & Mean \\
\hline $5 \times 5$ & 10.461 & 100.84 & 820 & 1220 & 1.95 & 2.263 \\
$9 \times 0$ & 10.872 & 102.84 & 768 & 1293 & 1.932 & 2.118 \\
$8 \times 8$ & 16.738 & 100.84 & 1312 & 1952 & 2.012 & 2.295 \\
$14 \times 0$ & 16.911 & 102.84 & 1344 & 1988 & 1.972 & 2.165 \\
$10 \times 10$ & 20.923 & 100.84 & 1640 & 2440 & 1.978 & 2.143 \\
$17 \times 0$ & 20.535 & 102.84 & 1728 & 2431 & 1.949 & 2.103 \\
$14 \times 14$ & 29.292 & 100.84 & 2296 & 3416 & 1.961 & 1.997 \\
$24 \times 0$ & 28.991 & 102.84 & 2304 & 3432 & 1.981 & 2.157 \\
$16 \times 16$ & 33.476 & 100.84 & 2624 & 3904 & 1.95 & 1.972 \\
$28 \times 0$ & 33.823 & 102.84 & 2784 & 4147 & 1.873 & 2.157 \\
\hline
\end{tabular}

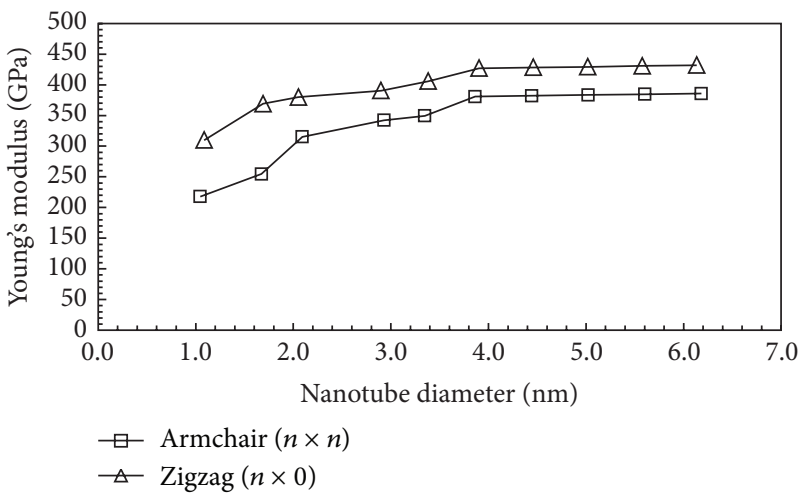

Figure 5: Effect of diameter on Young's modulus of SWZNTs.

having variations leading to depressions across the tube and in some cases less diameter at the ends. The ZNT is not a cylindrical tube as in CNT but it is irregular and is referred to as polygonal tube similar to other inorganic nanotubes [35]. The difference in geometrical orientation of ZNT compared to CNT is illustrated in Figure 4, both having the same number of bonds/elements and approximately the same diameter.

It has been established that some mechanical properties of CNTs are influenced by size and chirality [20,24]. Similar trend occurs in relation to ZNTs as indicated in Figure 5 showing changes of Young's modulus of armchair and zigzag nanotubes with diameter. The curve indicates significant effect of diameter on the value of $E$, especially in relation to small diameters. The zigzag ZNTs have higher Young's modulus in comparison with armchair ZNTs with similar diameters, but the pattern of increase is the same for all nanotubes. The increase in Young's modulus as the diameter increases is attributed to the effect of nanotube curvature $[26,36]$.

As the nanotube diameter increases, the effect of curvature reduces and $E$ converges to a value. For a variation of diameter from 1.05 to $6.18 \mathrm{~nm}$ for the armchair SWZNTs and from 1.09 to $6.13 \mathrm{~nm}$ for the zigzag SWZNTs, the values of

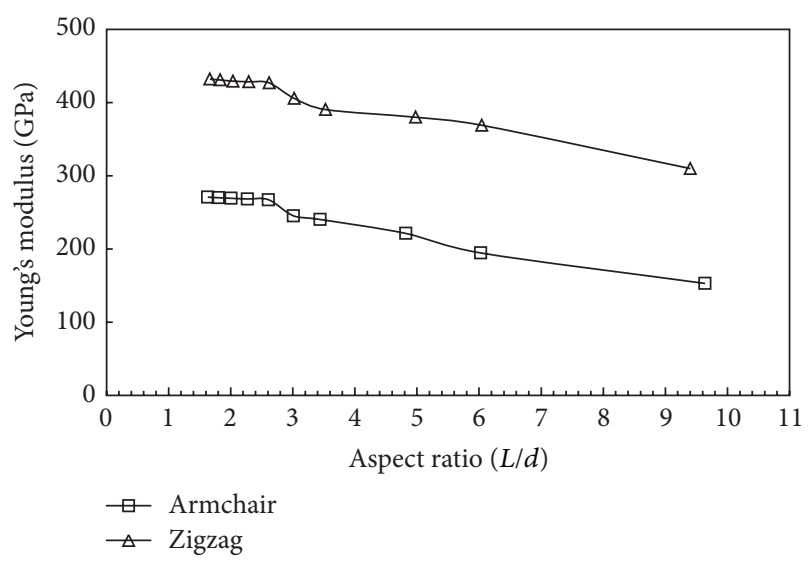

FIGURE 6: Effect of aspect ratio $(L / d)$ on Young's modulus of SWZNTs.

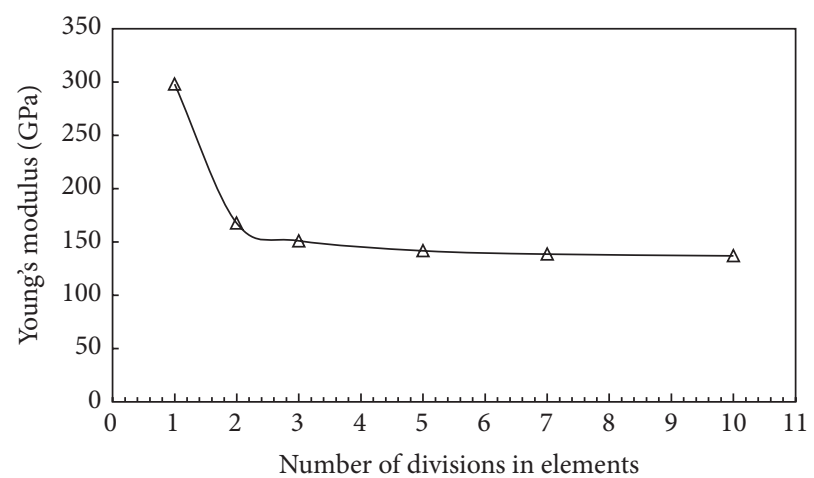

FIGURE 7: Curve obtained from convergence test for $(35 \times 0)$ SWZNT.

$E$ vary from 217 to $385 \mathrm{GPa}$ and from $309 \mathrm{GPa}$ to $431 \mathrm{GPa}$, respectively. The findings indicate that Young's moduli computed for both armchair and zigzag SWZNTs are constant for diameters ranging from 3.8 to $6.13 \mathrm{~nm}$ and are approximately 380 and $427 \mathrm{GPa}$ for armchair and zigzag, respectively. 


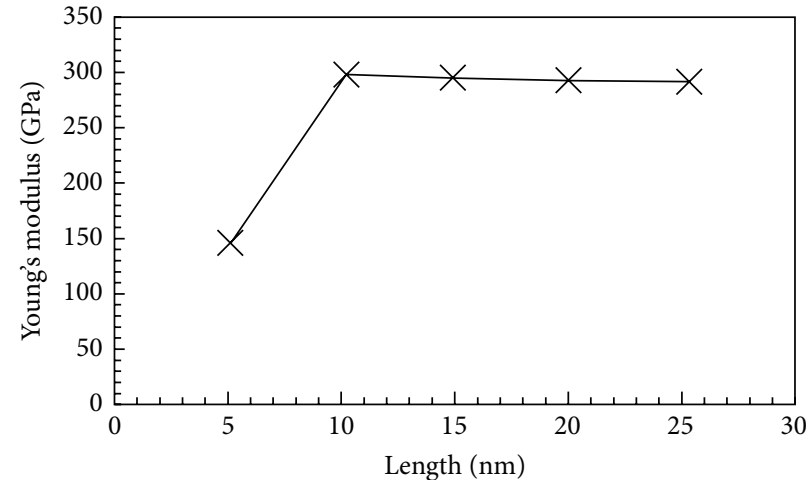

(a)

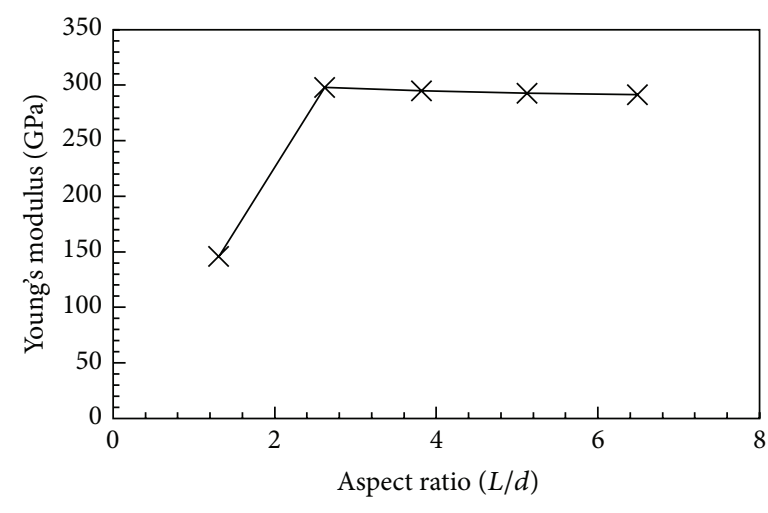

(b)

FIGURE 8: Variation of Young's modulus of $(35,0)$ SWZNT with (a) length and (b) aspect ratio.
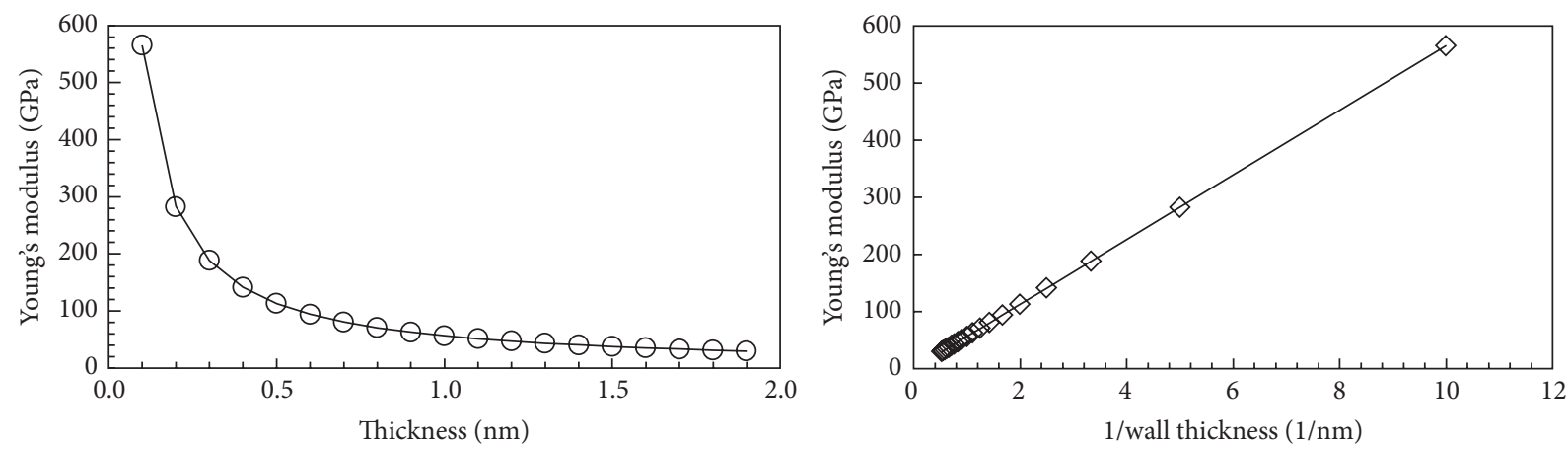

FIGURE 9: Effect of thickness on Young's modulus of $(35,0)$ SWZNT.

In contrast, Young's moduli of the SWZNTs decrease as the aspect ratio $(L / d)$ increases (Figure 6). This indicates that increasing the aspect ratio will negatively affect the structural stability of the nanotubes as in other inorganic nanotubes [26]. Thus, in relation to optimum Young's modulus, minimal diameter is required.

Based on minimal diameter, the SWZNT with optimal Young's modulus was found out to be the zigzag type with (35, 0 ) configuration as indicated in Figure 5. But Young's modulus of SWZNTs obtained is much higher than experimental and simulated values for inorganic nanotubes $[18,19,22,23]$. In order to obtain a more accurate solution, convergence test was conducted using h-method by creating finer mesh until the solution converges or approaches a particular value. From Figure 7, convergence occurred at 5 divisions of the element having Young's modulus as $141.64 \mathrm{GPa}$ compared to 297.96 GPa when division of the elements was 1 which is about $110 \%$ reduction. Dividing the elements further up to 10 units gave a difference of $3 \%$ from what was obtained for 5 divisions of the elements. The results obtained for the convergence test of $(35,0)$ SWZNT are shown in Figure 7.

With respect to length and aspect ratio, a similar pattern was observed for $(35 \times 0)$ SWZNT as illustrated in Figure 8 . There was tremendous increase in Young's modulus initially until the optimum value of $297 \mathrm{GPa}$ was attained at $10 \mathrm{~nm}$ length and aspect ratio of 2 and thereafter convergence occurred.

As illustrated in Figure 4, the ZNT is not a cylindrical tube but polygonal tube. Thus, the thickness of the tube is not assumed to be equivalent to the thickness of the bond/element as in CNT but is defined as difference of radial spaces between the furthest and innermost (oxygen) atoms in the optimized structures [25]. The wall thickness of SWZNT modelled from cubic nanosheet varies between 0.194 and $0.680 \mathrm{~nm}$ depending on symmetry and chirality [25]. As was obtained in CNT [36], the wall thickness has substantial influence on the computed Young's modulus as shown in Figure 9.

From the results obtained after simulation, it was noted that the greater the wall thickness of SWZNTs, the lesser the value of $E$ computed. For a variation of $t$ from $0.05 \mathrm{~nm}$ to $0.2 \mathrm{~nm}$, the value of $E$ varied from 565 to $67 \mathrm{GPa}$ for the $(35 \times 0)$ nanotube and from 431 to $53 \mathrm{GPa}$ for the $(20,20)$ nanotube. Thus, the result confirms what was obtained during nanoindentation of ZNT where thicker arrays were found out to be softer than their thinner equivalents [19].

It has been established that, for any tube wall thickness $t$ and chirality $(n \times m)$, there occurs a diameter $d$ below which 
the NTs develop instability and experience impulsive damage or destruction [25]. In order to maintain uniformity in the parametric studies of ZNT, the value of 0.194 was adopted for $t$ which is equivalent to the thickness of cubic zirconia nanosheet cleaved along (111) plane [27].

\section{Conclusions}

In this study, the tensile behavior of cubic single-walled zirconia nanotubes was simulated using nonlinear finite element analysis in order to establish geometrical parameters and magnitude of Young's modulus. The model was developed based on the assumption that ZNTs when subjected to load in form of strain behave as a space frame-like structure made up of elements connected by nodes. This allows linking the force constants in molecular mechanics and the elastic properties of the beam-like element member in structural mechanics through the energy equivalence theory. Simulations on nanotubes with different configurations were conducted by making one end fixed and subjecting the other end to axial tensile strain. The geometry of SWZNTs was found out to be polygonal tube not as cylindrical tube in CNT. The optimum Young's modulus of about $142 \mathrm{GPa}$ was obtained from zigzag SWZNT having diameter of about $6.15 \mathrm{~nm}$. Based on the results obtained, it can be concluded that the method used is an effective tool for investigating the mechanical properties of ZNTs and other nanotubes at less computational cost.

\section{Conflict of Interests}

The authors declare that there is no conflict of interests regarding the publication of this paper.

\section{Acknowledgments}

The authors are grateful for the support provided by Universiti Teknologi PETRONAS and Malaysian Ministry of Higher Education (MOHE) through the Long Term Research Grant Scheme (LRGS) for One Baja Research Programme (Project 6).

\section{References}

[1] M. C. Muñoz, S. Gallego, J. I. Beltrán, and J. Cerdá, "Adhesion at metal- $\mathrm{ZrO}_{2}$ interfaces," Surface Science Reports, vol. 61, no. 7, pp. 303-344, 2006.

[2] E. J. Walter, S. P. Lewis, and A. M. Rappe, "First principles study of carbon monoxide adsorption on zirconia-supported copper," Surface Science, vol. 495, no. 1-2, pp. 44-50, 2001.

[3] S. Meriani and C. Palmonari, Zirconia'88: Advances in Zirconia Science and Technology, Kluwer Academic, 1989.

[4] V. R. Choudhary, S. Banerjee, and S. G. Pataskar, "Combustion of dilute propane over transition metal-doped $\mathrm{ZrO}_{2}$ (cubic) catalysts," Applied Catalysis A: General, vol. 253, no. 1, pp. 6574, 2003.

[5] F. Rohr, P. Hagenmuller, and W. van Gool, Solid Electrolytes, Material Science Series, Academic Press, New York, NY, USA, 1978.
[6] A. Meldrum, L. A. Boatner, and R. C. Ewing, "Nanocrystalline zirconia can be amorphized by ion irradiation," Physical Review Letters, vol. 88, no. 2, Article ID 025503, 2001.

[7] M. Wilson, U. Schönberger, and M. W. Finnis, “Transferable atomistic model to describe the energetics of zirconia," Physical Review B, vol. 54, no. 13, pp. 9147-9152, 1996.

[8] M. Gateshki, V. Petkov, T. Hyeon, J. Joo, M. Niederberger, and Y. Ren, "Interplay between the local structural disorder and the length of structural coherence in stabilizing the cubic phase in nanocrystalline $\mathrm{ZrO}_{2}$," Solid State Communications, vol. 138, no. 6, pp. 279-284, 2006.

[9] Y. L. Soo, P. J. Chen, S. H. Huang et al., "Local structures surrounding $\mathrm{Zr}$ in nanostructurally stabilized cubic zirconia: structural origin of phase stability," Journal of Applied Physics, vol. 104, no. 11, Article ID 113535, 2008.

[10] T. Yamaguchi, M. Tan-No, and K. Tanabe, " $\mathrm{ZrO}_{2}$ as a catalyst and catalyst support," Journal of the Japan Petroleum Institute, vol. 36, no. 4, pp. 250-267, 1993.

[11] G. Cao, Nanostructures and Nanomaterials: Synthesis, Properties and Applications, Imperial College Press, London, UK, 2004.

[12] R. A. Evarestov, Y. F. Zhukovskii, A. V. Bandura, and S. Piskunov, "Symmetry and models of single-wall $\mathrm{BN}$ and $\mathrm{TiO}_{2}$ nanotubes with hexagonal morphology," Journal of Physical Chemistry C, vol. 114, no. 49, pp. 21061-21069, 2010.

[13] B. Wen Xing, Z. Chang Chun, and C. Wan Zhao, "Simulation of Young's modulus of single-walled carbon nanotubes by molecular dynamics," Physica B: Condensed Matter, vol. 352, no. 1-4, pp. 156-163, 2004.

[14] Y. Zhu, C. Ke, and H. D. Espinosa, "Experimental techniques for the mechanical characterization of one-dimensional nanostructures," Experimental Mechanics, vol. 47, no. 1, pp. 7-24, 2007.

[15] E. W. Wong, P. E. Sheehan, and C. M. Lieber, "Nanobeam mechanics: elasticity, strength, and toughness of nanorods and nanotubes," Science, vol. 277, no. 5334, pp. 1971-1975, 1997.

[16] M.-F. Yu, T. Kowalewski, and R. S. Ruoff, "Investigation of the radial deformability of individual carbon nanotubes under controlled indentation force," Physical Review Letters, vol. 85, no. 7, pp. 1456-1459, 2000.

[17] M.-F. Yu, O. Lourie, M. J. Dyer, K. Moloni, T. F. Kelly, and R. S. Ruoff, "Strength and breaking mechanism of multiwalled carbon nanotubes under tensile load," Science, vol. 287, no. 5453, pp. 637-640, 2000.

[18] T. Shokuhfar, G. K. Arumugam, P. A. Heiden, R. S. Yassar, and C. Friedrich, "Direct compressive measurements of individual titanium dioxide nanotubes," ACS Nano, vol. 3, no. 10, pp. 30983102, 2009.

[19] L.-N. Wang and J.-L. Luo, "Fabrication and mechanical properties of anodized zirconium dioxide nanotubular arrays," Journal of Physics D: Applied Physics, vol. 44, no. 7, Article ID 075301, 2011.

[20] C.-W. Fan, J.-H. Huang, C. Hwu, and Y.-Y. Liu, "Mechanical properties of single-walled carbon nanotubes-a finite element approach," Advanced Materials Research, vol. 33-37, pp. 937942, 2008.

[21] C. Wang, Multiscale modeling and simulation of nanocrystalline zirconium oxide [Ph.D. thesis], University of Nebraska, 2009.

[22] E. Kalfon-Cohen, O. Goldbart, R. Schreiber et al., "Radial compression studies of $\mathrm{WS}_{2}$ nanotubes in the elastic regime," Journal of Vacuum Science and Technology B: Microelectronics and Nanometer Structures, vol. 29, no. 2, Article ID 021009, 2011. 
[23] T. Lorenz, D. Teich, J.-O. Joswig, and G. Seifert, "Theoretical study of the mechanical behavior of individual $\mathrm{TiS}_{2}$ and $\mathrm{MoS}_{2}$ nanotubes," Journal of Physical Chemistry C, vol. 116, no. 21, pp. 11714-11721, 2012.

[24] X. Chen and G. Cao, "A structural mechanics study of singlewalled carbon nanotubes generalized from atomistic simulation," Nanotechnology, vol. 17, no. 4, pp. 1004-1008, 2006.

[25] A. V. Bandura and R. A. Evarestov, "Ab initio structure modeling of $\mathrm{ZrO}_{2}$ nanosheets and single-wall nanotubes," Computational Materials Science, vol. 65, pp. 395-405, 2012.

[26] R. Ansari, S. Rouhi, M. Mirnezhad, and F. Sadeghiyeh, "Studying the buckling and vibration characteristics of single-walled zinc oxide nanotubes using a nanoscale finite element model," Applied Physics A: Materials Science and Processing, vol. 112, no. 3, pp. 767-774, 2013.

[27] L. Boldrin, F. Scarpa, R. Chowdhury, and S. Adhikari, "Effective mechanical properties of hexagonal boron nitride nanosheets," Nanotechnology, vol. 22, no. 50, Article ID 505702, 2011.

[28] L. Guimarães, A. N. Enyashin, G. Seifert, and H. A. Duarte, "Structural, electronic, and mechanical properties of singlewalled halloysite nanotube models," Journal of Physical Chemistry C, vol. 114, no. 26, pp. 11358-11363, 2010.

[29] Y. Mitsunori and I. T. Yuko, "Synthesis and applications of zirconia and ruthenium oxide nanotubes," in Inorganic and Metallic Nanotubular Materials, T. Kijima, Ed., pp. 117-133, Springer, Berlin, Germany, 2010.

[30] I. D. Muhammad, M. Awang, O. Mamat, and Z. B. Shaari, "First-principles calculations of the structural, mechanical and thermodynamics properties of cubic zirconia," World Journal of Nano Science and Engineering, vol. 4, no. 2, pp. 97-103, 2014.

[31] I. D. Muhammad and M. Awang, "Extracting the atomic coordinates and connectivity of zirconia nanotubes from PDB files for modelling in ANSYS," Advances in Nanoparticles, vol. 3, pp. 92-98, 2014.

[32] “4.188 BEAM188 3-D Linear Finite Strain Beam,” September 2014, http://mostreal.sk/html/elem_55/chapter4/ES4-188.htm.

[33] R. A. Evarestov, Y. F. Zhukovskii, A. V. Bandura, and S. Piskunov, "Symmetry and models of single-wall $\mathrm{BN}$ and $\mathrm{TiO}_{2}$ nanotubes with hexagonal morphology," The Journal of Physical Chemistry C, vol. 114, no. 49, pp. 21061-21069, 2010.

[34] D. Dass, R. Prasher, and R. Vaid, "Analytical study of unit cell and molecular structures of single walled carbon nanotubes," International Journal of Computational Engineering Research, vol. 2, pp. 1447-1457, 2012.

[35] K. Tibbetts, R. Doe, and G. Ceder, "Polygonal model for layered inorganic nanotubes," Physical Review B-Condensed Matter and Materials Physics, vol. 80, no. 1, Article ID 014102, 2009.

[36] K. I. Tserpes and P. Papanikos, "Finite element modeling of single-walled carbon nanotubes," Composites Part B: Engineering, vol. 36, no. 5, pp. 468-477, 2005. 

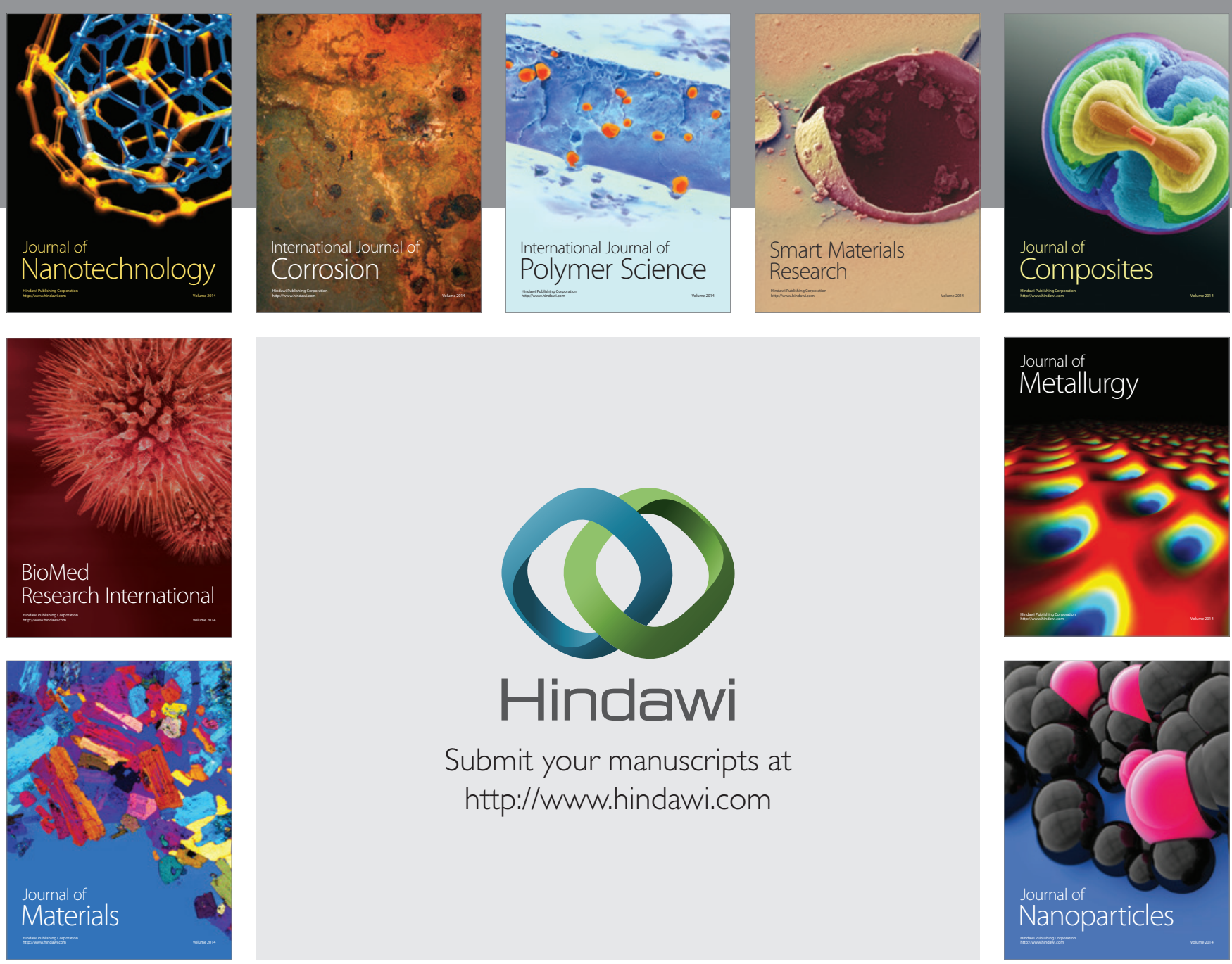

Submit your manuscripts at http://www.hindawi.com
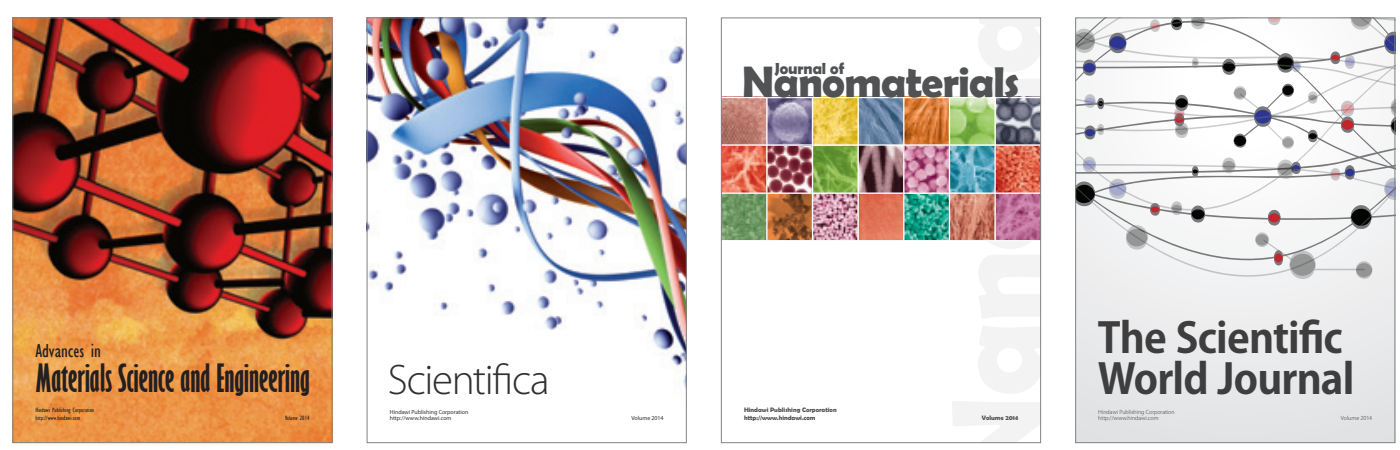

\section{The Scientific World Journal}
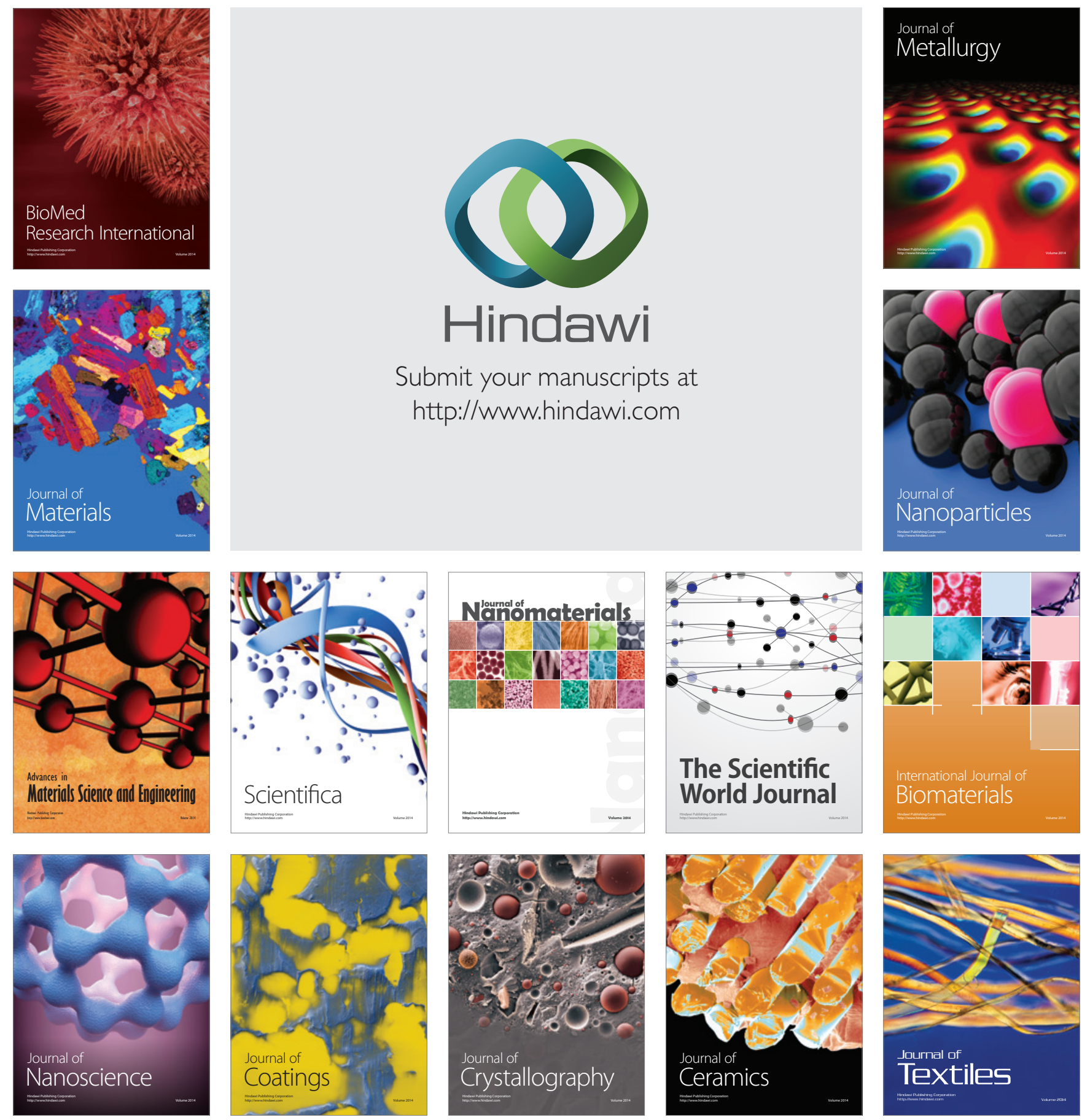